\title{
KHOVANOV HOMOLOGY DETECTS THE HOPF LINKS
}

\author{
JOHN A. BALDWIN, STEVEN SIVEK, AND YI XIE
}

\begin{abstract}
We prove that any link in $S^{3}$ whose Khovanov homology is the same as that of a Hopf link must be isotopic to that Hopf link. This holds for both reduced and unreduced Khovanov homology, and with coefficients in either $\mathbb{Z}$ or $\mathbb{Z} / 2 \mathbb{Z}$.
\end{abstract}

Khovanov homology [Kho00] associates to each link $L \subset S^{3}$ a bigraded group $K h^{*, *}(L)$, whose graded Euler characteristic recovers the Jones polynomial $V_{L}(q)$, as well as a reduced variant $K h_{\text {red }}^{* * *}(L)$ [Kho03. It is known to detect the unknot [KM11, the $n$-component unlink for all $n$ [BS15, and the trefoils [BS18]. In this note we prove the same for the Hopf links $H_{ \pm}$, which are oriented so that the two components have linking number \pm 1 . Then

$$
\begin{aligned}
& K h\left(H_{+} ; \mathbb{Z}\right) \cong \mathbb{Z}_{(0,0)} \oplus \mathbb{Z}_{(0,2)} \oplus \mathbb{Z}_{(2,4)} \oplus \mathbb{Z}_{(2,6)} \\
& K h\left(H_{-} ; \mathbb{Z}\right) \cong \mathbb{Z}_{(0,0)} \oplus \mathbb{Z}_{(0,-2)} \oplus \mathbb{Z}_{(-2,-4)} \oplus \mathbb{Z}_{(-2,-6)}
\end{aligned}
$$

where $\mathbb{Z}_{(h, q)}$ is a copy of $\mathbb{Z}$ in bigrading $(h, q)$. The reduced Khovanov homology of a link depends on a choice of distinguished component, which we generally suppress from the notation, but we have $K h_{\text {red }}\left(H_{+} ; \mathbb{Z}\right) \cong \mathbb{Z}_{(0,1)} \oplus \mathbb{Z}_{(2,5)}$ and $K h_{\text {red }}\left(H_{-} ; \mathbb{Z}\right) \cong \mathbb{Z}_{(0,-1)} \oplus \mathbb{Z}_{(-2,-5)}$ regardless of this choice.

Theorem 1. Let $L$ be a link in $S^{3}$ such that either $K h(L) \cong K h\left(H_{ \pm}\right)$or $K h_{\mathrm{red}}(L) \cong$ $K h_{\text {red }}\left(H_{ \pm}\right)$as bigraded groups, with coefficients in either $\mathbb{Z}$ or $\mathbb{Z} / 2 \mathbb{Z}$. Then $L=H_{ \pm}$.

Our proof makes use of a handful of spectral sequences involving Khovanov homology. Batson and Seed's link splitting spectral sequence [BS15], together with Kronheimer and Mrowka's spectral sequence converging to singular instanton knot homology [KM11, will tell us that such a link $L$ must have exactly two unknotted components with linking number \pm 1 . Once we know the module structure on $K h_{\text {red }}(L)$, we apply a refinement by the third author [Xie18] of the latter spectral sequence to determine that the instanton knot homology $K H I(L)$ of [KM10b] has rank at most 4. We will then determine the Alexander grading on $K H I(L)$ to see that $L$ has Seifert genus zero, and conclude that $L$ must be a Hopf link.

If $L$ is a link with $r$ components, then $K h^{*, *}(L)$ is invariant as a module over the ring

$$
R_{r}=\mathbb{Z}\left[x_{1}, \ldots, x_{r}\right] /\left\langle x_{1}^{2}, \ldots, x_{r}^{2}\right\rangle
$$

Kho03, HN13, in which each $x_{i}$ preserves the $h$ ("homological") grading while lowering the $q$ ("quantum") grading. If we define the reduced Khovanov homology of $L$ using the component which corresponds to $x_{r}$, then $K h_{\text {red }}^{*, *}(L)$ is a module over $R_{r-1}$. We begin by determining this module structure.

Proposition 2. Let $L$ be a link in $S^{3}$, and let $H$ be either $H_{+}$or $H_{-}$. Let $\mathbb{F}=\mathbb{Z} / 2 \mathbb{Z}$. Suppose that any one of the following is true as an isomorphism of bigraded groups:

(1) $K h(L ; \mathbb{Z}) \cong K h(H ; \mathbb{Z})$.

JAB was supported by NSF CAREER Grant DMS-1454865. 
(2) $K h(L ; \mathbb{F}) \cong K h(H ; \mathbb{F})$.

(3) $K h_{\text {red }}(L ; \mathbb{Z}) \cong K h_{\text {red }}(H ; \mathbb{Z})$ for some choice of component of $L$.

(4) $K h_{\text {red }}(L ; \mathbb{F}) \cong K h_{\text {red }}(H ; \mathbb{F})$ for some choice of component of $L$.

Then $L$ has exactly two components, which are both unknots, and if $H=H_{ \pm}$then their linking number is \pm 1 . Moreover, we have $\operatorname{rank} K h_{\text {red }}(L ; \mathbb{Z})=2$, with

$$
K h_{\mathrm{red}}(L ; \mathbb{Q}) \cong \begin{cases}\mathbb{Q}_{(0,1)} \oplus \mathbb{Q}_{(2,5)}, & H=H_{+} \\ \mathbb{Q}_{(0,-1)} \oplus \mathbb{Q}_{(-2,-5)}, & H=H_{-},\end{cases}
$$

and the $R_{1}$-action on $K h_{\mathrm{red}}(L ; \mathbb{Q})$ is trivial.

Proof. Suppose that $H=H_{+}$; the case of $H_{-}$is identical. We first claim that each of conditions (11), (2), and (3) implies condition (4), and then use the latter to prove the rest of the proposition. Certainly (11) implies (2) and (3) implies (4) by the universal coefficient theorem. Moreover, (2) and (4) are equivalent by the identity

$$
K h^{h, q}(K ; \mathbb{F}) \cong K h_{\text {red }}^{h, q-1}(K ; \mathbb{F}) \oplus K h_{\text {red }}^{h, q+1}(K ; \mathbb{F})
$$

where $K$ is an arbitrary link [Shu14, Corollary 3.2.C], so this proves the claim.

We suppose from now on that condition (44) holds; since this is equivalent to (2), we have

$$
\begin{aligned}
K h(L ; \mathbb{F}) & \cong \mathbb{F}_{(0,0)} \oplus \mathbb{F}_{(0,2)} \oplus \mathbb{F}_{(2,4)} \oplus \mathbb{F}_{(2,6)} \\
K h_{\text {red }}(L ; \mathbb{F}) & \cong \mathbb{F}_{(0,1)} \oplus \mathbb{F}_{(2,5)} .
\end{aligned}
$$

If $L$ were a knot, then the rank of $K h_{\text {red }}(L ; \mathbb{F})$ would be congruent $\bmod 2$ to $V_{L}(-1)=$ $\pm \operatorname{det}(L) \equiv 1(\bmod 2)$, so $L$ must be a link, say $L=K_{1} \cup K_{2} \cup \cdots \cup K_{r}$ with $r \geq 2$.

We apply the rank inequality [BS15, Corollary 1.6] derived from Batson and Seed's link splitting spectral sequence, namely that if $\mathbb{K}$ is any field then

$$
\operatorname{rank} K h(L ; \mathbb{K}) \geq \operatorname{rank} \bigotimes_{i=1}^{r} K h\left(K_{i} ; \mathbb{K}\right) .
$$

For $\mathbb{K}=\mathbb{F}$, the left side is 4 while the right side is at least $2^{r}$ with equality if and only if $K_{i}$ is unknotted [KM11, so $r=2$ and $K_{1}$ and $K_{2}$ are both unknotted. (See BS15, Proposition 7.1].) In fact, this inequality respects the grading $\ell=h-q$ : if we let $t=$ $2 \mathrm{lk}\left(K_{1}, K_{2}\right)$, then [BS15, Corollary 4.4] says that

$$
\operatorname{rank}^{\ell} K h(L ; \mathbb{F}) \geq \operatorname{rank}^{\ell+t}\left(\bigotimes_{i} K h\left(K_{i} ; \mathbb{F}\right)\right)=\operatorname{rank}^{\ell+t}\left(\mathbb{F}_{(0,-1)} \oplus \mathbb{F}_{(0,1)}\right)^{\otimes 2}
$$

The left side has ranks $1,2,1$ in $\ell$-gradings $0,-2,-4$ respectively, and the right side has rank 2 when $\ell+t=0$, so we must have $-2+t=0$, or $\operatorname{lk}\left(K_{1}, K_{2}\right)=\frac{1}{2} t=1$.

Finally, applying the inequality (1) with $\mathbb{K}=\mathbb{Q}$ says that $\operatorname{rank} K h(L ; \mathbb{Q}) \geq 4$, and there is an exact triangle

$$
\cdots \rightarrow K h(L ; \mathbb{Q}) \rightarrow K h_{\text {red }}(L ; \mathbb{Q}) \rightarrow K h_{\text {red }}(L ; \mathbb{Q}) \rightarrow \ldots,
$$

so we must have $\operatorname{rank} K h_{\text {red }}(L ; \mathbb{Q}) \geq 2$. Applying the universal coefficient theorem repeatedly, we determine first that $\operatorname{rank} K h_{\text {red }}(L ; \mathbb{Z}) \geq 2$, and that if this inequality is strict then $\operatorname{rank} K h_{\text {red }}(L ; \mathbb{F})>2$ as well, which is false. Thus $\operatorname{rank} K h_{\text {red }}(L ; \mathbb{Z})=2=\operatorname{rank} K h_{\text {red }}(L ; \mathbb{F})$, 
and so the $\mathbb{Z}$ summands of the former have the same bigrading as the $\mathbb{F}$ summands of the latter. We deduce from this that

$$
K h_{\text {red }}(L ; \mathbb{Q}) \cong \mathbb{Q}_{(0,1)} \oplus \mathbb{Q}_{(2,5)} .
$$

Now if we pick a distinguished component of $L$ so that $K h_{\text {red }}(L ; \mathbb{Q})$ is a module over $R_{1}=$ $\mathbb{Z}\left[x_{1}\right] /\left\langle x_{1}^{2}\right\rangle$, then $x_{1}$ acts with square zero on each $K h_{\text {red }}^{h, *}(L ; \mathbb{Q})$, which is either 0 or $\mathbb{Q}$, and so it must act trivially as claimed.

We will now make use of Kronheimer and Mrowka's instanton knot homology KHI, defined in [KM10b] and extended to links in [KM10a. They showed in KM11 that for any knot $K$, the complex vector space $K H I(K)$ is isomorphic to the reduced singular instanton knot homology $I^{\natural}(K ; \mathbb{C})$, and using a spectral sequence $K h(\bar{K}) \Rightarrow I^{\natural}(K)$ they deduced a rank inequality

$$
\operatorname{rank} K h_{\text {red }}(K) \geq \operatorname{rank} K H I(K) \text {. }
$$

Based on results in $\mathrm{KM} 10 \mathrm{~b}$, this proved that $K h_{\text {red }}$ has rank 1 if and only if $K$ is unknotted. The third author Xie18 extended this from knots to pointed links and incorporated the module structure on Khovanov homology to prove the following.

Theorem 3 ([Xie18, Theorem 5.4]). Let $L$ be a link of $r$ components in $S^{3}$, and fix a base point on the rth component, equipping $K h_{\mathrm{red}}(L ; \mathbb{Z})$ with an $R_{r-1}$-module structure. Let $X^{\prime}=\left(x_{1}, \ldots, x_{r-1}\right) \in R_{r-1}^{\oplus r-1}$. Then

$$
\operatorname{rank} K H I(L) \leq \operatorname{rank} H^{*}\left(K h_{\text {red }}(L ; \mathbb{Z}) \otimes_{R_{r-1}} K\left(X^{\prime}\right)\right),
$$

where $K\left(X^{\prime}\right)$ is the Koszul complex

$$
0 \rightarrow R_{r-1} \stackrel{\wedge X^{\prime}}{\longrightarrow} \Lambda^{1} R_{r-1}^{\oplus r-1} \stackrel{\wedge X^{\prime}}{\longrightarrow} \Lambda^{2} R_{r-1}^{\oplus r-1} \stackrel{\wedge X^{\prime}}{\longrightarrow} \ldots \stackrel{\wedge X^{\prime}}{\longrightarrow} \Lambda^{r-1} R_{r-1}^{\oplus r-1} \rightarrow 0 .
$$

Proposition 4. Let $L \subset S^{3}$ be a link satisfying any of the hypotheses of Proposition Q. Then $\operatorname{rank} K H I(L) \leq 4$.

Proof. Proposition 2 tells us that $L$ is a 2-component link, and that $K h_{\text {red }}(L ; \mathbb{Q}) \cong \mathbb{Q}^{2}$ has a trivial action of $R_{1}=\mathbb{Z}\left[x_{1}\right] /\left\langle x_{1}^{2}\right\rangle$. In this case the Koszul complex $K\left(X^{\prime}\right)$ is

$$
0 \rightarrow R_{1} \stackrel{\cdot x_{1}}{\longrightarrow} R_{1} \rightarrow 0
$$

and $K h_{\text {red }}(L ; \mathbb{Q}) \cong\left(R_{1} /\left\langle x_{1}\right\rangle \otimes_{\mathbb{Z}} \mathbb{Q}\right)^{\oplus 2}$ as $R_{1}$-modules. The complex

$$
\left(R_{1} /\left\langle x_{1}\right\rangle \otimes_{\mathbb{Z}} \mathbb{Q}\right) \otimes_{R_{1}} K\left(X^{\prime}\right)=(0 \rightarrow \mathbb{Q} \stackrel{0}{\rightarrow} \mathbb{Q} \rightarrow 0)
$$

has cohomology $\mathbb{Q}^{2}$, so we conclude that

$$
\operatorname{rank} H^{*}\left(K h_{\text {red }}(L ; \mathbb{Z}) \otimes_{R_{1}} K\left(X^{\prime}\right)\right)=4
$$

and the proposition now follows from Theorem 3 .

For links in $S^{3}$ (and more generally for null-homologous links with a choice of Seifert surface), instanton knot homology is equipped with an Alexander grading

$$
K H I(L)=\bigoplus_{j=-g+1-r}^{g-1+r} K H I(L, j),
$$


where $L$ has $r$ components and Seifert genus $g$. This grading is symmetric in the sense that $K H I(L, j) \cong K H I(L,-j)$ for all $j$. Each $K H I(L, j)$ is canonically $\mathbb{Z} / 2 \mathbb{Z}$-graded, and the Euler characteristics of these summands determine the Alexander polynomial of $L$ by

$$
\sum_{j} \chi(K H I(L, j)) t^{j}=-\left(t^{1 / 2}-t^{-1 / 2}\right)^{r-1} \Delta_{L}(t) .
$$

See [KM10a, Theorem 3.6] or [Lim10].

Let $\tilde{\Delta}_{L}\left(t_{1}, t_{2}\right)$ denote the multivariable Alexander polynomial of a 2-component link $L=K_{1} \cup K_{2}$. This is related to the single-variable Alexander polynomial $\Delta_{L}(t)$ by

$$
\Delta_{L}(t)= \pm\left(t^{1 / 2}-t^{-1 / 2}\right) \tilde{\Delta}_{L}(t, t),
$$

see e.g. [Mil68, Lemma 10.1]. It follows from (2) that

$$
\sum_{j} \chi(K H I(L, j)) t^{j}= \pm\left(t^{1 / 2}-t^{-1 / 2}\right)^{2} \tilde{\Delta}_{L}(t, t),
$$

and hence that the right side of

$$
\tilde{\Delta}_{L}(t, t)= \pm\left(\frac{\sum_{j} \chi(K H I(L, j)) t^{j}}{t-2+t^{-1}}\right)
$$

must be a Laurent polynomial, i.e. that $t-2+t^{-1}$ divides the numerator. Torres Tor53] proved that $\tilde{\Delta}_{L}\left(t_{1}, t_{2}\right)$ also satisfies $\tilde{\Delta}_{L}(1,1)= \pm \operatorname{lk}\left(K_{1}, K_{2}\right)$.

Proposition 5. Let $L=K_{1} \cup K_{2}$ be a two-component link with $\operatorname{lk}\left(K_{1}, K_{2}\right)= \pm 1$. If rank $K H I(L) \leq 4$, then

$$
K H I(L)=\mathbb{C}_{1} \oplus\left(\mathbb{C}^{\oplus 2}\right)_{0} \oplus C_{-1},
$$

where the subscripts denote the Alexander grading, and $\Delta_{L}(t)= \pm\left(t^{1 / 2}-t^{-1 / 2}\right)$.

Proof. We first claim that in fact $K H I(L)$ is nonzero in at least three different Alexander gradings. If not, then (3) has the form

$$
\tilde{\Delta}_{L}(t, t)= \pm\left(\frac{c_{1} t^{e_{1}}+c_{2} t^{e_{2}}}{t-2+t^{-1}}\right)
$$

for some integers $c_{1}, c_{2}$ which are not both zero and some integers $e_{1} \neq e_{2}$. (The numerator cannot be identically zero because then $\tilde{\Delta}_{L}(t, t)=0$, contradicting $\tilde{\Delta}_{L}(1,1)= \pm 1$.) The denominator has a double root at $t=1$, so it cannot divide the numerator, which is either a monomial (and is thus nonzero when $t=1$ ) or a binomial with only simple roots, and we have a contradiction.

Next, we show that $\operatorname{rank} K H I(L)=4$. It is already at least three, since it has positive rank in each of at least three Alexander gradings. But the rank must be even, since it has the same parity as $\sum_{j} \chi(K H I(L, j))(-1)^{j}$ and this sum is equal to $\pm 4 \tilde{\Delta}_{L}(-1,-1) \in 4 \mathbb{Z}$ by setting $t=-1$ in (3). Thus the total rank is 4 as claimed.

We let $m>k$ be the two largest Alexander gradings in which $K H I(L)$ is nonzero. By symmetry $-m$ is the lowest such grading, so $-m<k<m$; then $K H I(L,-k) \neq 0$ as well, so we must have had $k \geq 0$. If $k>0$ then $\operatorname{KHI}(L, j)$ has positive rank for each of $j= \pm m, \pm k$, and its total rank is 4 , so it must have rank exactly one in each of these gradings. Otherwise $k=0$, and by symmetry we have $\operatorname{rank}(K H I(L)) \equiv \operatorname{rank}(K H I(L, 0))(\bmod 2)$; the former 
is even, so the latter is as well, and $\operatorname{KHI}(K, j)$ now has rank at least $1,2,1$ for each of $j=m, 0,-m$ and total rank 4 . In either case we conclude that

$$
K H I(L) \cong \mathbb{C}_{m} \oplus \mathbb{C}_{k} \oplus \mathbb{C}_{-k} \oplus \mathbb{C}_{-m}
$$

where $m>k \geq 0$.

We do not know the $\mathbb{Z} / 2 \mathbb{Z}$ grading of each summand, but from (3) there must be signs $\epsilon_{m}, \epsilon_{k}, \epsilon_{-k}, \epsilon_{-m} \in\{ \pm 1\}$ such that

$$
\tilde{\Delta}_{L}(t, t)= \pm\left(\frac{\epsilon_{m} t^{m}+\epsilon_{k} t^{k}+\epsilon_{-k} t^{-k}+\epsilon_{-m} t^{-m}}{t-2+t^{-1}}\right) .
$$

The numerator must be a multiple of $(t-1)^{2}$, so both it and its derivative are zero at $t=1$, giving us the conditions

$$
\epsilon_{m}+\epsilon_{k}+\epsilon_{-k}+\epsilon_{-m}=0, \quad m\left(\epsilon_{m}-\epsilon_{-m}\right)+k\left(\epsilon_{k}-\epsilon_{-k}\right)=0 .
$$

From the second equation we have $\epsilon_{m}=\epsilon_{-m}$, or else the first term would have magnitude $2 m$ and thus be strictly greater than $\left|k\left(\epsilon_{k}-\epsilon_{-k}\right)\right| \leq 2 k$; and from $\sum \epsilon_{j}=0$ we now have $\epsilon_{k}+\epsilon_{-k}=-2 \epsilon_{m}$, so that $\epsilon_{k}=\epsilon_{-k}=-\epsilon_{m}$. We conclude that

$$
\tilde{\Delta}_{L}(t, t)= \pm\left(\frac{t^{m}-t^{k}-t^{-k}+t^{-m}}{t-2+t^{-1}}\right) .
$$

Finally, we use the fact that $\tilde{\Delta}_{L}(1,1)= \pm 1$ to determine $m$ and $k$. Given an equation involving Laurent polynomials of the form $p(t)=t^{c}(t-1)^{2} q(t)$ we have $q(1)=\frac{1}{2} p^{\prime \prime}(1)$, and so taking $p(t)= \pm\left(t^{m}-t^{k}-t^{-k}+t^{-m}\right)$ and $q(t)=\tilde{\Delta}_{L}(t, t)$ gives us

$$
\tilde{\Delta}_{L}(1,1)= \pm\left.\frac{1}{2} \frac{d^{2}}{d t^{2}}\left(t^{m}-t^{k}-t^{-k}+t^{-m}\right)\right|_{t=1}= \pm\left(m^{2}-k^{2}\right) .
$$

Since $m>k \geq 0$, this can only be equal to \pm 1 if $m=1$ and $k=0$. Thus $K H I(L)$ is exactly as claimed, while $\tilde{\Delta}_{L}(t, t)= \pm 1$ and $\Delta_{L}(t)= \pm\left(t^{1 / 2}-t^{-1 / 2}\right) \tilde{\Delta}_{L}(t, t)= \pm\left(t^{1 / 2}-t^{-1 / 2}\right)$.

We now know enough about any $\operatorname{link} L$ with the same Khovanov homology as a Hopf link $H_{ \pm}$to determine its link type.

Proof of Theorem 1. We have $L=K_{1} \cup K_{2}$, with both $K_{i}$ unknotted and $\operatorname{lk}\left(K_{1}, K_{2}\right)= \pm 1$, by Proposition 2. Proposition 4 gives us the bound $\operatorname{rank} K H I(L) \leq 4$, so then

$$
K H I(L) \cong \mathbb{C}_{1} \oplus\left(\mathbb{C}^{\oplus 2}\right)_{0} \oplus \mathbb{C}_{-1}
$$

by Proposition 5, where again the subscripts denote the Alexander grading.

We now claim that the Seifert genus of $L$ is 0 , as a consequence of the more general

$$
g(L)+r-1=\max \{j \mid K H I(L, j) \neq 0\}
$$

for $r$-component links in $S^{3}$ with irreducible complement. This generalizes the case $r=1$ of [KM10b, Proposition 7.16], and the proof is essentially the same. If $\Sigma$ is a genus- $g$ Seifert surface for $L$, then we identify the appropriate Alexander grading with a sutured instanton homology group,

$$
K H I(L, g+r-1) \cong S H I\left(S^{3}(\Sigma)\right),
$$

where $S^{3}(\Sigma)$ is obtained by cutting open the complement of $L$ (with a pair of meridional sutures on each component) along $\Sigma$. (The reason for the shift by $r-1$ is that the $j$ th Alexander grading is the generalized $2 j$-eigenspace of an operator $\mu(\bar{\Sigma})$ on the instanton 
homology of some closed manifold, and the maximal real eigenvalue of $\mu(\bar{\Sigma})$ is $2 g(\bar{\Sigma})-2=$ $2(g+r-1)$, as in [KM10a, $\S 2.5]$.) If $\Sigma$ is genus-minimizing then $S^{3}(\Sigma)$ is a taut sutured manifold, so $S H I\left(S^{3}(\Sigma)\right) \neq 0$ by [KM10b, Theorem 7.12] and the claim follows.

Thus $L$ bounds an annulus $A$, and the core of $A$ is isotopic to either of the unknots $K_{i}$ on its boundary. The boundary is in particular a cable of the unknot, and the only such 2-component links with linking number \pm 1 are the positive and negative Hopf links.

\section{REFERENCES}

[BS15] Joshua Batson and Cotton Seed. A link-splitting spectral sequence in Khovanov homology. Duke Math. J., 164(5):801-841, 2015.

[BS18] John A. Baldwin and Steven Sivek. Khovanov homology detects the trefoils. arXiv:1801.07634, 2018.

[HN13] Matthew Hedden and Yi Ni. Khovanov module and the detection of unlinks. Geom. Topol., 17(5):3027-3076, 2013.

[Kho00] Mikhail Khovanov. A categorification of the Jones polynomial. Duke Math. J., 101(3):359-426, 2000 .

[Kho03] Mikhail Khovanov. Patterns in knot cohomology. I. Experiment. Math., 12(3):365-374, 2003.

[KM10a] Peter Kronheimer and Tom Mrowka. Instanton Floer homology and the Alexander polynomial. Algebr. Geom. Topol., 10(3):1715-1738, 2010.

[KM10b] Peter Kronheimer and Tomasz Mrowka. Knots, sutures, and excision. J. Differential Geom., 84(2):301-364, 2010.

[KM11] P. B. Kronheimer and T. S. Mrowka. Khovanov homology is an unknot-detector. Publ. Math. Inst. Hautes Études Sci., (113):97-208, 2011.

[Lim10] Yuhan Lim. Instanton homology and the Alexander polynomial. Proc. Amer. Math. Soc., 138(10):3759-3768, 2010.

[Mil68] John Milnor. Singular points of complex hypersurfaces. Annals of Mathematics Studies, No. 61. Princeton University Press, Princeton, N.J.; University of Tokyo Press, Tokyo, 1968.

[Shu14] Alexander N. Shumakovitch. Torsion of Khovanov homology. Fund. Math., 225(1):343-364, 2014.

[Tor53] Guillermo Torres. On the Alexander polynomial. Ann. of Math. (2), 57:57-89, 1953.

[Xie18] Yi Xie. Earrings, sutures and pointed links. arXiv:1809.09254, 2018.

E-mail address: john.baldwin@bc.edu

Department of Mathematics, Boston College

E-mail address: s.sivek@imperial.ac.uk

Department of Mathematics, Imperial College London

E-mail address: yxie@scgp.stonybrook.edu

Simons Center for Geometry and Physics, State University of New York 